\title{
Proposal of a Method for Promotion of Continuous Pro-Environmental Behavior with Easy Communication
}

\author{
Saizo Aoyagi, Tomoaki Okamura, Hirotake Ishii, and Hiroshi Shimoda \\ Graduate School of Energy Science, Kyoto University, Yoshida-honnmachi, \\ Sakyo-ku, Kyoto 606-8501, Japan \\ \{aoyagi, okamura, hirotake, shimoda\} @ei.energy.kyoto-u.ac.jp
}

\begin{abstract}
In Japan, Promotion of Domestic Pro-Environmental Behavior(PEB) is one of the main challenges for energy saving. Existing studies of Promotion of PEB seldom deal with continue of PEB. Purposes of this study are proposal of a method for promotion of continuous PEB with easy communication and evaluation of the effectiveness of the method. Main part of the proposed method is an easy communication system to cause social facilitation of PEB among users. An evaluation experiment with ten participants was conducted and the result showed that the proposed method promotes domestic PEB if "feeling of joint PEB" is aroused.
\end{abstract}

Keywords: Pro-Environmental Behavior, Computer Mediated Communication, Social impact theory, Easy communication, Social stress.

\section{Introduction}

In Japan, energy consumption of residential sector has risen because both number of households and households energy consumption have increased. As a result, energy saving in this sector is recognized as one of pressing issues by the government [1]. Domestic Pro-Environmental Behavior (PEB) of people is a concrete way of energy saving in residential sector and there have been many studies about factors which promote PEB of people [2,3] and methods of promotion of that [4-6]. Most studies in this area have just deal promotion of PEB, and few studies [7] definitely focused on continue of PEB despite of its importance. Promotion of starting of PEB of people has no meaning if they give up PEB easily.

Purposes of this study are proposal of a method for promotion of continuous PEB with easy communication and evaluation of the effectiveness of the method.

\section{Method for Promotion of Continuous Pro-Environmental Behavior}

\subsection{Approach and Requirements of the Proposed Method}

A study said that habit formation of an action needs continuance of that action about a month [9]. Based on the study, we intend to continue PEB of target people of the 
method until habit formation of PEB, and the method is designed to be used for a month, not endlessly used.

Next, this study employed socio-psychological social facilitation [8] effect as a main principle for promoting continuous PEB. Social facilitation means that people conform their action to others. This effect works on people each other, and will form feedback loops of PEB; social facilitation therefore will be useful to promote continuous PEB.

\section{- Stress-free communication about PEB}

Nevertheless, some study said that people recently do not like conventional deep or close communication, because of social stress associated with it and stress-free is main point of continue communication $[10,11]$. Recent development of twitter, very easy way of communication supports these studies [12]. This is a problem because knowing the fact that others do PEB is essential to cause social facilitation and it is conventionally associated with communication. Consequently, stress-free and easy communication method for PEB promotion is necessary.

- Feeling of joint PEB in the same "room"

Nevertheless, it is afraid that easy and stress-free communication causes weaker social facilitation because social impact can be a kind of social stress. Therefore, social facilitation caused by easy communication is desired to be as storing as other requirements allow.

\section{- Secure existence of others doing PEB}

Next, it is possible that the feedback loop of social facilitation of PEB is broken when a user feel that others don't do PEB by some chance. Consequently, a measure for avoiding break of the feedback loop of social facilitation of PEB is needed.

\section{- Awareness of PEB occasion}

At last, according to cognitive psychology, people's actions in daily life are embedded in time-series schema and implemented following schemas in each situation without being intended [14]. Therefore, if someone does not do PEB in daily life, occasions of PEB in daily life do not surface to the conscious mind and are likely let past. Consequently, some way to show occasions of PEB to people who do not do PEB in daily life in order to promote PEB of them.

\subsection{Details of the Proposed Method}

We propose a method for promotion of continuous PEB. The proposed method employs an easy communication system which uses portable multimedia device or Smartphone, its client software, web server and server software. Users of the system communicate in a group and are promoted their continuous PEB. The system has following functions. Screenshots of the proposed system are shown in fig 1 .

- Easy communication method

Main functions of the system are two easy communication methods, "mutter" and "PEB footprints" which clear some kinds of social stress for sending information about PEB which was done. 
"Mutter" is a limited text-based Computer Mediated Communication (CMC) method shown in right part of fig. 1. It has no destination address; it therefore cannot be use for one-on-one communication in order to free users from obligation of reply and expectation of replies, which can be a social-stress. In addition, the number of characters of mutter is limited up to 140 in order to free users from obligation of writing "good text" with caring about others' eyes.

"PEB footprint" is another CMC method shown in left part of fig. 1.

It can be sent through one-touch operation, and expresses only the fact that someone does a PEB by fixed sentence for each PEB. Therefore users need not write text by themselves in order to free users from obligation of writing "good text" with caring about others' eyes.

- Room-specific timeline

Next, "room-specific timeline" displays others' mutters and PEB footprints to a user shown in left part of fig. 1. According to social impact theory [13], immediacy affects impact of social facilitation. Based on this theory, "room-specific timeline" promotes social facilitation effects through strengthening spatial immediacy artificially and arouse a kind of feeling of users. They will feel that they do PEB together in the same "room" when they view room-specific timeline of the place they are in that time. This effect is named as "feeling of joint PEB in the same room". This feeling do not mean that people think physically occupy the same place. "Room" in this context is just an imaginings.

- Conversational agent

In addition, "Conversational agent" promotes formation of positive feedback loop of social facilitation through behaving as "others who do PEB". Conversational agents always behave as artificial active people who do PEB in order to avoid such situations. It is implemented with artificial intelligence technologies or acted by people who are in charge of promotion of PEB of people.

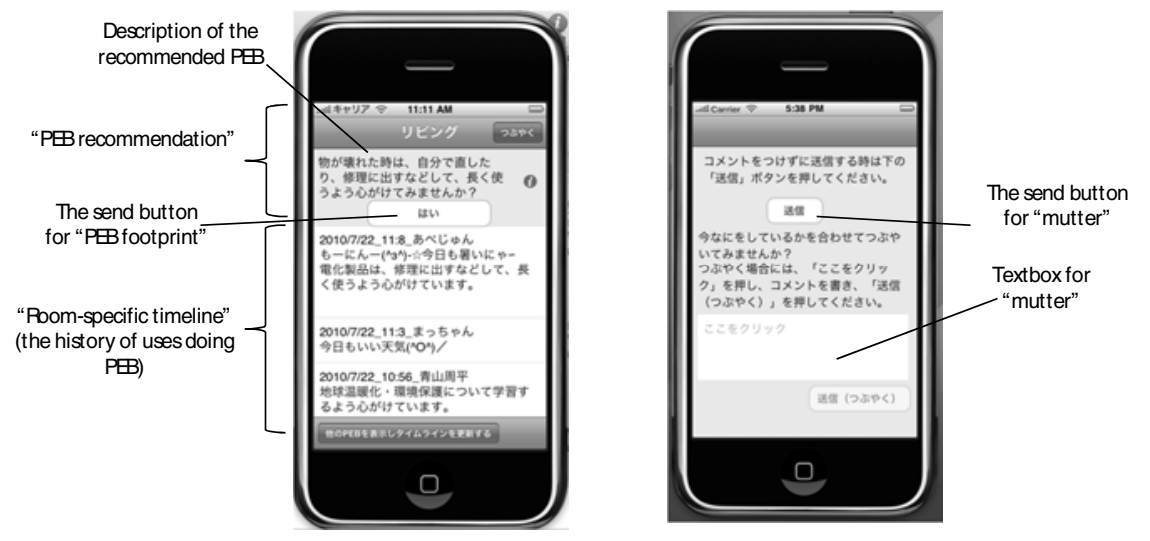

Fig. 1. Screenshots of the proposed method 


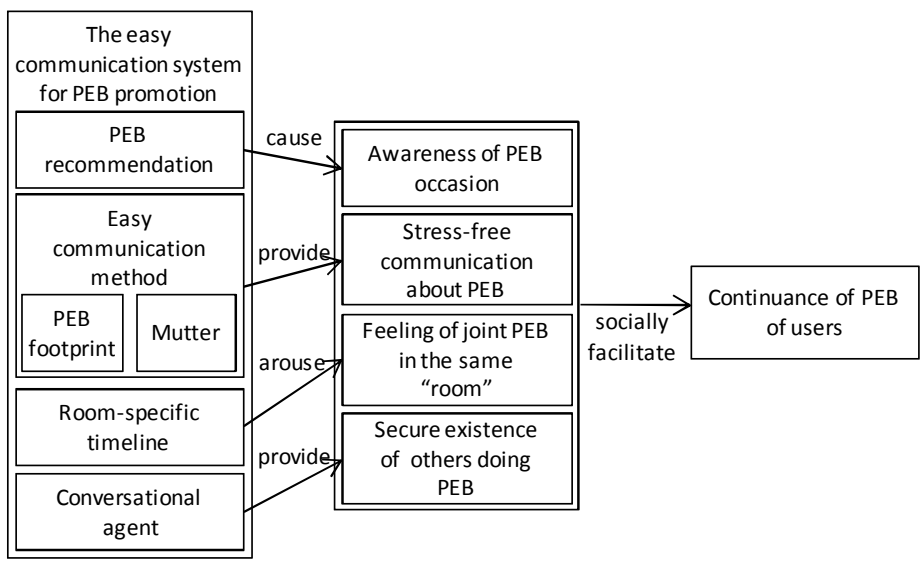

Fig. 2. A framework of the proposed system

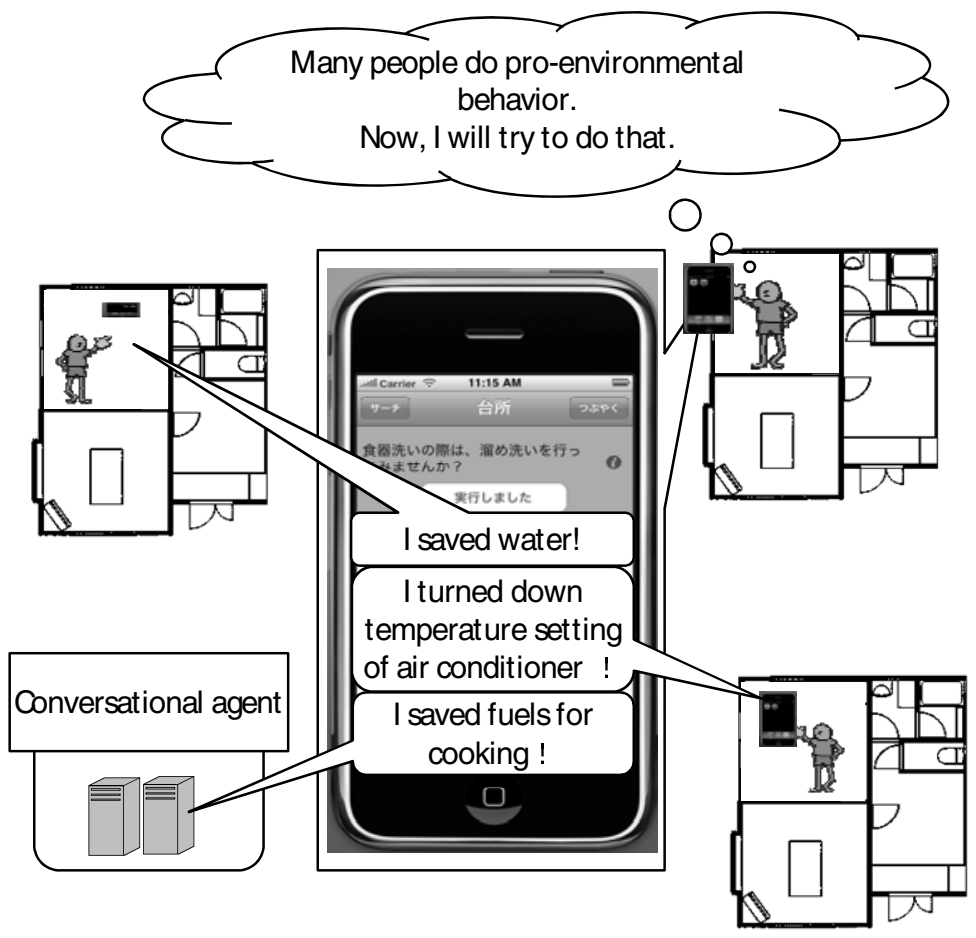

Fig. 3. An example of how the proposed system works

- PEB recommendation

At last "PEB recommendation" gives awareness of occasions of PEB of people based on time schedule of their daily life so as avoid oversight of the occasions. It shows an available PEB in that time to users as shown in left part of fig. 1. 
Correspondences between requirements and functions are shown in fig. 2.

Figure 3 shows an example of how the proposed system works and how users use it. As this figure shows, users carry around smartphone with the client software of the system in their home, and announce the fact that they do PEB to other users with "PEB footprints" function of the system. "PEB footprints" are shown to other users by "room specific timeline" functions of the system in displays of smartphone. "PEB footprints" are sometimes sent by conversational agents in order to avoid break of the feedback loop of social facilitation. If some users view many "PEB footprints" in "room specific timeline", they will intend to do PEB by social facilitation effect.

\section{Evaluation of the Proposed System}

\subsection{Purpose}

An evaluation experiment was conducted for evaluation of effectiveness of the proposed system through checking following two points. First is whether feeling of joint PEB is aroused and second is promotion of continuous PEB by feeling of joint PEB.

\subsection{Method}

In the experiment, some participants actually used the system in their households, and the period of use in the experiment was a month from 2010 November 15 to December 14. Participants of the experiment were two groups. The younger group consists of five 20's people and the elder group consists of four 50's people and a 60 person. In addition, each group includes five conversational agents. Each participant used an iPod touch which client software of the system was installed in. Each iPod touch was connected to server software of the system through Wi-Fi station.

Before the period of use, a questionnaire was conducted to gather information about participants' daily life pattern, frequency of PEB, and other basic information of them. In the period of use, participants use the client software in iPod touch and communicate with other group members, 58 kinds of PEB were recommended based on their daily life patterns. In addition, they answer questionnaires about frequency of PEB for the last week, which were recommended by the system once a week. After the period, a questionnaire was conducted to gather participants ' subjective evaluation of starting, increasing frequency, and continuing of PEB through use of the system, and subjective evaluation of functions of the system, such as whether they felt others doing PEB in the same "room" through use of the system. Participants got ten thousand Japanese yen after all activity in the experiment were finished. They were told about this reward before the period, they however were not told amount of money.

Log data of the system, such as number of PEB footprints of each participant were recorded in the period. This log data and answers of questionnaire were collected in order to evaluation of the system from multiple points of view.

\subsection{Results and Discussion}

All participants answered all questionnaires and log data was collected without problems. Table 1 shows results of questionnaire on effectiveness of system functions. Y1-Y5 mean participants in the younger group and E1-E5 mean participants in the 
elder group. This table shows that five participants felt (answered 4 or 5) others doing PEB in the same "room" and remaining participants felt others doing PEB not in the same "room". Moreover, most participants didn't feel (answered 1 or 2) four socialstress associated with communication, "minded others", "expected replies", "felt obligated to reply", "felt obligated to reply to reply". These results suggest effectiveness of functions of the easy communication system.

Table 1. Results of questionnaire on effectiveness of system functions

\begin{tabular}{lllllll}
\hline Participant & $\begin{array}{l}\text { Felt others } \\
\text { doing PEB } \\
\text { in the same } \\
\text { "room" }\end{array}$ & $\begin{array}{l}\text { Felt others } \\
\text { doing } \\
\text { PEB** }\end{array}$ & $\begin{array}{l}\text { Minded } \\
\text { others }\end{array}$ & $\begin{array}{l}\text { Expected } \\
\text { replies }\end{array}$ & $\begin{array}{l}\text { Felt } \\
\text { obligated } \\
\text { to reply }\end{array}$ & $\begin{array}{l}\text { Felt } \\
\text { obligated } \\
\text { to reply } \\
\text { to reply }\end{array}$ \\
\hline Y1 & 5 & & 1 & 2 & 4 & 2 \\
Y2 & 2 & 5 & 1 & 3 & 2 & 3 \\
Y3 & 2 & 4 & 1 & 1 & 1 & 1 \\
Y4 & 4 & & 2 & 1 & 1 & 1 \\
Y5 & 2 & 5 & 2 & 4 & 4 & 3 \\
E1 & 2 & 3 & 4 & 1 & 1 & 1 \\
E2 & 5 & & 1 & 3 & 4 & 2 \\
E3 & 1 & 4 & 1 & 1 & 3 & 1 \\
E4 & 4 & & 2 & 4 & 2 & 2 \\
E5 & 4 & & 2 & 2 & 2 & 3 \\
\hline
\end{tabular}

* Answered with number (5: I completely agree - 1: I completely disagree)

**: Participant who did not felt others doing PEB in the same "room" only.

Figure 4 shows frequency of PEB of participants. Participants answered "always", "often", "sometimes", "occasionally", "not at all" and "no occasion" about 58 PEBs to questionnaires which measures frequency of PEB. PEBs which were not answered as "no occasion" through the period by each participant were shown in fig. 4 for comparison. Figure 4 shows that frequencies of PEBs had increased basically.

Nevertheless, there were some exceptions. For example, PEB frequencies of Y1, $\mathrm{Y} 3, \mathrm{Y} 4, \mathrm{E} 1, \mathrm{E} 4$, and E5 once decreased from the questionnaire before the period to the questionnaire a week after, and increased after that. These results are explained by difference between questionnaire before the period and other questionnaire of PEB frequency. The questionnaire before the period is considered to be answered by guess because people were assumedly not conscious of their behaviors in daily life, other questionnaires however are considered to be answered based on credible memories because people were conscious of PEB being affected this experiment. Consequently we mainly use results of questionnaires from a week after to a month after, and results of the questionnaire before the period are used as reference.

Next, Kruskal-Wallis tests were conducted in order to evaluate promotion of PEB through the period and table 2 shows significance of change of PEB frequency through the period. Considering the phenomenon described above, Kruskal-Wallis tests were conducted (1) between answer of the questionnaire before the period and that of a month after, and (2) between that of a week after and that of a month after. Table 2 shows that five participants had increased PEB frequency significantly through the period and three participants in them had evidently increased PEB frequency. 

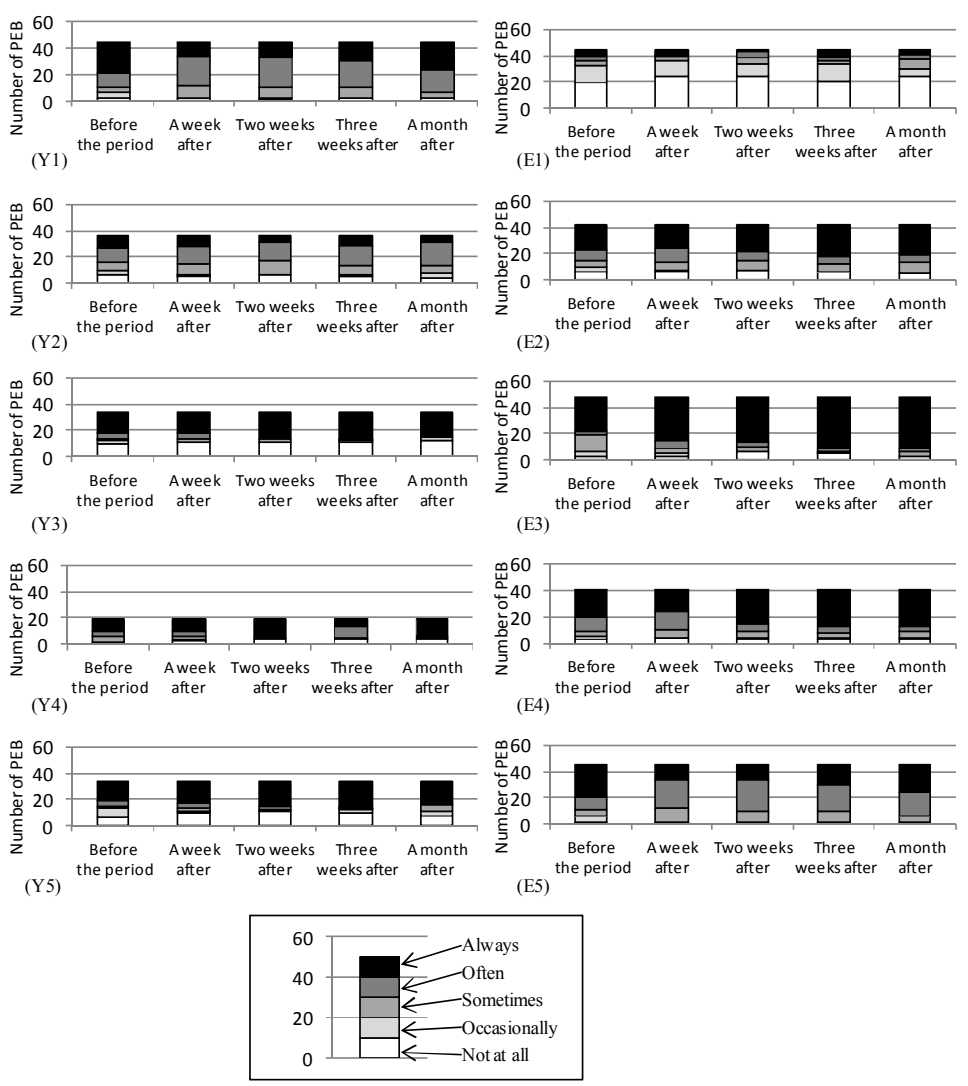

Fig. 4. Results of questionnaire on frequency of PEB

Figure 5 shows the number of "PEB footprints" in the experimental period. PEB footprints of participants showed decreasing trend, they however continued through the experimental period. It is evidence which suggests effectiveness of the system.

Next, table 3 shows subjective evaluation of starting, increasing frequency, and continuing of PEB through use of the system. This table shows that four participants began new PEB, six people increased PEB frequency, and five participants continue PEB subjectively.

To summarize, fig. 2, table 2 and table 3 suggest that PEB of about a half of all participants were promoted and continued a month.

There are some differences between results of questionnaire on PEB frequency shown in fig. 2 and subjective evaluation after a month and shown in table 3. For example, E3 could not begin new PEB, increased PEB frequency, and continued PEB subjectively, he/she however show significant increase of PEB frequency. Nevertheless, they are considered to be caused by lapse of memory or change of baseline of evaluation, which are common thing to human.

At last, we found a factor which affected promotion of PEB and continuous PEB in comparison between table 1 and table 3.Table 1 showed that Y1, Y4, E2, E4, and E5 felt (answered 4 or 5) others doing PEB in the same "room" and the same participants 

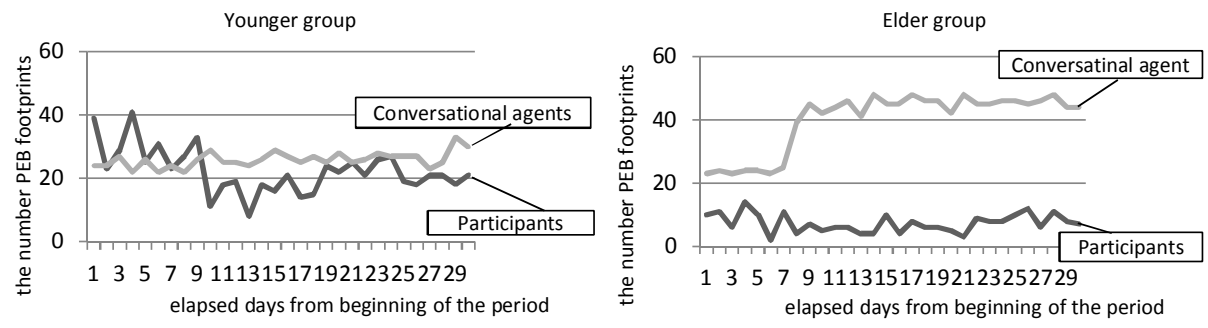

Fig. 5. The number of "PEB footprints" in the experimental period

Table 2. Significance of change of PEB frequancy between two questionnaires

\begin{tabular}{lll}
\hline Participants & $\begin{array}{l}\text { Before the period and } \\
\text { a month after }\end{array}$ & $\begin{array}{l}\text { A week after and } \\
\text { A month after }\end{array}$ \\
\hline Y1 & n.s. & $*$ \\
Y2 & n.s. & n.s. \\
Y3 & n.s. & n.s. \\
Y4 & n.s. & n.s. \\
Y5 & n.s. & n.s. \\
E1 & n.s. & n.s. \\
E2 & $*$ & n.s. \\
E3 & $* *$ & n.s. \\
E4 & n.s. & $* *$ \\
E5 & n.s. & $*$ \\
\hline$*:$ p $<0.05, * *: p<0.01$. &
\end{tabular}

Table 3. Subjective evaluation of starting, increasing frequency, and continuing of PEB through use of the system

\begin{tabular}{llll}
\hline Participant & $\begin{array}{l}\text { Began new } \\
\text { PEB }\end{array}$ & $\begin{array}{l}\text { Increased } \\
\text { PEB fre- } \\
\text { quency }\end{array}$ & $\begin{array}{l}\text { Continued } \\
\text { PEB }\end{array}$ \\
\hline Y1 & 3 & 5 & 5 \\
Y2 & 3 & 4 & 2 \\
Y3 & 1 & 3 & 2 \\
Y4 & 4 & 5 & 4 \\
Y5 & 1 & 2 & 2 \\
E1 & 2 & 2 & 2 \\
E2 & 5 & 4 & 4 \\
E3 & 2 & 2 & 2 \\
E4 & 4 & 4 & 4 \\
E5 & 4 & 4 & 4 \\
\hline
\end{tabular}

* answered with number (5: I completely agree - 1: I completely disagree). 
also increased continued PEB (answered 4 or 5) in table 3 . In addition, about the same participants increased PEB and begun PEB (answered 4 or 5). These results suggest feeling of joint PEB in the same "room" is a key factor to promote continuous PEB and only feeling of joint PEB (not in the same "room") is not sufficient.

\section{Summary and Future Task}

In this study, the method for promotion of continuous PEB with easy communication was proposed and it was evaluated through the experiment. The results showed that continuous PEB of some participants were promoted by the proposed method.

Nevertheless, other participants however not continued, and suggested that feeling of joint PEB in the same "room" is a key factor to promote continuous PEB. In this regard, however feeling of joint PEB was achieved in remaining participants. This result suggests that remaining assignments for us is to enhance immediacy which is a factor of strengthening social facilitation effect. In other word, future task is to enhance feeling of being in the same "room".

\section{References}

1. METI: Annual Energy Report 2010 (2010)

2. Hirose, Y.: Determinants of Environment-Conscious Behavior. Social Psychology 10(1), 44-55 (1994)

3. Kollmus, A., Agyeman, J.: Mind the Gap: why do people act environmentally and what are the barriers to pro-environmental behavior? Environmental Education Research 8(3), 239260 (2002)

4. Abrahamse, W., Steg, L., Vlek, C., Rothengatter, T.: A review of Intervention Studies Aimed to Household Energy Conservation. Journal of Environmental Psychology 25, 273 291 (2005)

5. Ohta, K., Fujii, S.: An Experimental Study on Effects of Providing Objective Information about Reduction of $\mathrm{CO}_{2}$ Emission due to Pro-Environmental Behavior. Journals of the Japan Society of Civil Engineers G. 63(2), 159-168 (2007)

6. Matsumura, N.: A study on Effects of Role Playing on Behavioral Change toward Environmental Conservation. In: Proceedings of Infrastructure Planning, vol. 27, p. 144 (2003)

7. Tan, Y.: Persuasive Technology in Motivating Household Energy Conservation. In: Michaelles, F. (ed.) Business Aspects of the Internet of Things Seminar of Advanced Topics. FS 2009, pp. 52-58 (2009)

8. Moscovici, S., Personnaz, B.: Studies in Social Influence. Journal of Experimental Social Psychology 16, 270-282 (1980)

9. Sharma, R.: Who Will Cry When You die? HarperCollins, Canada (2000)

10. Sato, I.: Communications of "shyness" in dance: body presentation of "I" in club. Journal of Communication Studies 27, 51-72 (2007)

11. Muramoto, Y.: Fuzzy Boundary between Group and Togetherness Situation: A Variety of Identity Found at a Park in Every Morning. Japanese Journal of Social 12(2), 112-124 (1996)

12. Twitter, http: / /www. twitter.com

13. Latane, B.: The Psychology of Social Impact. American Psychologist 3(4), 343-356 (1981)

14. Schank, R.C., Abelson, R.P.: Scripts, Plans, Goals, and Understanding: An Inquiry into Human Knowledge Structures. The Halsted Press Division of John Wiley and Sons, New York (1977) 